ARTíCULOS 



\title{
Aclaraciones sobre la mejora humana
}

\author{
Explanations on human enhancement
}

FRANCISCO JAVIER LÓPEZ FRÍAS*

\begin{abstract}
Resumen: En este artículo se pretende arrojar algo de luz al debate en torno a la mejora humana. Para ello, pretenderá ofrecer una definición ajustada de qué podemos considerar como «mejora» y, tras ello, analizará cuáles son las intervenciones técnicas que se incluirían bajo dicho concepto.
\end{abstract}

Palabras clave: mejora humana, bioética, terapia.
Abstract: This paper shall try to shed light on the human enhancement debate. In order to do so, it shall propose an accurate definition of the term «enhancement». After so doing, it shall analyze the type of interventions that could be included within such a definition.

Key words: human enhancement, bioethics, therapy.

\section{La mejora humana: reto ético de nuestro tiempo}

La idea de mejorar la especie humana ha estado siempre presente a lo largo de la Historia. No obstante, sería la Ilustración la que comenzaría a profundizar de forma más directa en ella. Como es bien sabido, intervenciones como la educación moral y la racionalización del Estado se concibieron como elementos esenciales para el progreso y la liberación de los seres humanos. No hay más que recordar las prácticas de los déspotas ilustrados o, a nivel teórico, el famoso texto kantiano «¿Qué es la Ilustración?», en el que éste afirma:

«La Ilustración es la liberación del hombre de su culpable minoría de edad. La minoría de edad significa la incapacidad de servirse de su inteligencia sin la guía de otro.

Fecha de recepción: 03/12/2012. Fecha de aceptación final: 29/03/2014.

* Becario de Investigación en el Departamento de Filosofía Moral de la Universidad de Valencia. Realiza su tesis sobre mejora humana y filosofía práctica del deporte bajo la dirección del profesor Jesús Conill Sancho. Es miembro de diversas asociaciones filosóficas como la Asociación Internacional de Filosofía del Deporte (IAPS) y la Asociación Latina de Filosofía del Deporte (ALFiD). Ha realizado estancia de investigación en el Uehiro Centre for Practical Ethics de la Universidad de Oxford, la Penn State University, y la Universidad de Roma «Foro Itálico». Entre las publicaciones más destacadas: «El debate en torno a la mejora humana «sin etiquetas»» en Sistema: Revista de Ciencias Sociales y «Continuidad de las innovaciones tecnológicas: el reto de las intervenciones biomédicas de mejora humana» en Isegoría.

Este trabajo se inscribe dentro del proyecto de investigación con referencia FFI2008-06133/FISO financiado por el Ministerio de Ciencia e Innovación. Becario de Investigación FPU (AP2009-4405) del Ministerio de Educación y Ciencia. Departamento de Filosofía del Derecho, Moral y Política. Universidad de Valencia. 
Y esa incapacidad es culpable porque su causa no reside en la falta de inteligencia sino de decisión y de valor para servirse por uno mismo de ella sin tutela ajena ${ }^{1}{ }_{»}$.

El éxito de la ciencia moderna fue central para este ideal de mejorar y empoderar a la naturaleza humana. Si en el siglo XVIII la matemática y la física eran las ciencias referentes, en el S. XIX la biología tomó el testigo. Sobre todo, tras la aparición de la teoría de la evolución de Darwin. Con su predominio, la idea de mejorar la naturaleza humana se biologizó, apareciendo con ello propuestas eugenésicas basadas en el darwinismo social. El mismo Nietzsche y su superhombre son una muestra clara de esta biologización del intento de mejorar la naturaleza humana ${ }^{2}$.

No obstante, los recientes avances de la ciencia genética, las neurociencias, y la biomedicina han provocado un giro radical en torno a esta cuestión. Ya no es que el hombre trate de disponer las cosas de modo que los seres humanos cambien en relación a lo externo; por medio de la educación, la selección en función de ciertas cualidades, la competencia, ciertos valores superiores, etc. Sino que el ser humano está por primera vez en su Historia capacitado para intervenir directamente en su propia naturaleza:

«Los seres humanos siempre han tratado de mejorarse a sí mismos — para incrementar sus capacidades mentales, física, y emocionales-. La invención de la escritura, por ejemplo, fue una mejora importante de nuestros poderes cognitivos [...] Pero por primera vez poseemos un conocimiento científico que tiene la capacidad de transformarnos a nosotros mismos, quizás, de un modo más profundo

$-\mathrm{y}$, de seguro, mucho más deliberado- que nunca antes» ${ }^{3}$

Estas nuevas técnicas plantean posibilidades que hace apenas unos años considerábamos propias de ciencia ficción: implantes de microchips en el cerebro que mejoran nuestra memoria, inyecciones de material genético que llevan nuestras capacidades físicas hasta límites nunca vistos, drogas que cambian nuestro modo de, en el sentido más heideggeriano, «estar en el mundo» volviéndonos más empáticos, más felices, más optimistas... Por ello, muchos auguran una revolución tan importante como la que llevó a cabo Galileo al «poner» a la Tierra a girar alrededor del Sol, o como la que provocó Darwin al «bajarnos a la Tierra» convirtiéndonos en parte de un eslabón evolutivo que, comenzando por partículas minúsculas aparecidas en el ámbito marino, desembocó en nosotros pasando por el mundo de los reptiles y los homínidos. Por ejemplo, el sociólogo Jeremy Rifkin asegura que seremos capaces de alcanzar un «Segundo Edén» ${ }^{4}$.

Ante tales posibilidades técnicas y promesas utópicas, el debate bioético no podía tardar demasiado en emerger con fuerza. A pesar de que las cuestiones morales que suscita no sean de extrema urgencia - como sí lo son otros que ya sufrimos actualmente como la pobreza,

1 I. KANT: «Respuesta a la pregunta ¿Qué es la Ilustración?» en J. B. ERHARD, J. G. Herder, I. KANT, ET AL: ¿Qué es la Ilustración?, trad. por Agapito Maestre, Tecnos, Madrid, 1988, p.17.

2 K. BAYERTZ: GenEthics: technological intervention in human reproduction as a philosophical problema, Cambridge, Cambridge University Press, 1994, cap. 1.

3 A. Buchanan: Beyond humanity? The ethics of biomedical enhancement, Oxford, Oxford University Press, p. xi.

4 J. RIFKIN: The Biotech Century, London, Phoenix, 1999. 
la desigualdad entre el Norte y el Sur, las injusticias sociales_-, la sola posibilidad de que estas técnicas estén dentro de un horizonte futuro nos exige, tal y como afirma Jonathan Glover, analizarlas seriamente para «pensar sobre el futuro por el que consideramos que merece la pena luchar» ${ }^{5}$.

Antes de afrontar tal reto moral, este artículo se plantea como objetivo ofrecer una panorámica global del problema, prestando principal atención a tres cuestiones: a) las dificultades que encontramos a la hora de definir la mejora (\$2), b) qué es aquello que podemos denominar como «mejora» $(\$ 3), c)$ el estado actual y la naturaleza de aquellas técnicas que se incluyen bajo dicha definición $(\S 4)$.

Autores como Julian Savulescu, Allen Buchanan o John Harris defienden un concepto amplio de la mejora, en el que caben elementos tan dispares como: una idea, el Estado, la alfabetización, la agricultura, un gen modificado artificialmente, o el último producto de la nano-robótica. Por el contrario, yo pretendo aportar una definición más acotada del mismo, restringida a intervenciones de carácter biomédico — aún a riesgo de caer en lo que Buchanan denomina como «excepcionalismo biotecnológico».

\section{2. ¿Qué es la mejora humana? Dificultades para encontrar un concepto}

Este apartado mostrará las principales dificultades con que nos encontramos a la hora de tratar de definir el concepto «mejora». Algunas de ellas son endémicas del debate, es decir, propias de las diferencias que existen entre los diversos autores y aproximaciones teóricas. Sin embargo, otras están arraigadas en errores lingüístico-conceptuales, como por ejemplo, el modo ingenuo y convencional en el que entendemos el concepto «mejora». Por otro lado, también podemos encontrar otras dificultades provocadas por la comprensión que realizamos de otros conceptos limítrofes al de mejora como «terapia», «naturaleza humana», $\mathrm{y}$ «enfermedad», entre otros.

Podría elaborarse todo un análisis completo sobre los intereses y prejuicios que están detrás de estas dificultades, sin embargo, este no es el objetivo de esta sección, cuya principal tarea es, simplemente, la de mostrar lo complejo que supone el ofrecer una definición del concepto «mejora», suponiendo que tal cosa sea posible, pues esto no es algo que todos acepten dentro de este debate — como se indicará más adelante. Muchos han obviado estas dificultades a la hora de establecer una definición de «mejora», el conocerlas a fondo nos ayudará a realizar esta tarea satisfactoriamente.

\subsection{Primera dificultad: Mejora, supuesta mejora e intervención}

El término «mejora» podría entenderse de un modo convencional, es decir, como lo define el diccionario: «adelantar, acrecentar algo, haciéndolo pasar a un estado mejor». La mejora humana sería, pues, la posibilidad de acrecentar o incrementar nuestros cuerpos, mentes y habilidades con el fin de llevarnos a un estado mejor. ¿Se refiere realmente este

5 J. Glover: What Sort of People Should There Be? Genetic engineering, brain control and their impact on our future world, New York, Penguin Books, 1984, p. 15; [trad. esp. El hombre prefabricado: problemas éticos de la ingeniería genética, Barcelona, Ariel, 1986]. 
debate ético sobre la mejora a todos los tipos de intervenciones que pueden incrementar de algún modo las capacidades y habilidades humanas? No parece que esto sea así. Muchos descubrimientos técnicos nos han permitido incrementar nuestras habilidades, como por ejemplo, los ordenadores o internet, sin embargo, ninguno de ellas ha generado un debate ético tan importante como el que discutiremos aquí — esto pone en duda la validez de la definición amplia de mejora, que concibe a ésta como cualquier invención humana que ha servido para llevar a un estado mejor sus condiciones de vida.

Además, el concepto «mejora» puede entenderse de modo ingenuo si se toma de un modo literal, pues si bien el propio término, como algunos autores transhumanistas han defendido, indica algo positivo: mejorar es alcanzar un estado más satisfactorio. No queda nada claro que todas aquellas intervenciones que se englobarán bajo este término sean verdaderas mejoras de la condición humana. Es más, debería hablarse siempre de supuestas mejoras —o de búsqueda de mejora, al menos, hasta que el debate bioético aclare de qué modo estas intervenciones de corte biotecnológico pueden servir al ser humano para vivir mejor. A lo largo de la Historia, siempre ha habido intervenciones con las que el hombre ha tratado de mejorar las condiciones con las que se encontraba, pero ni todas ellas fueron mejoras como tal, por ejemplo, la decisión de lanzar la bomba atómica sobre Hiroshima y Nagasaki, ni todas pueden incluirse en un todo, llamado «mejora humana» o «técnicas de mejora humana», que aceptemos o rechacemos de forma global ¿por qué no hablar de «peoras» 0 «empeoramiento»? .

Por lo tanto, ha de quedar claro que, primero, «mejora» debe distinguirse del concepto «intervención». Segundo, que por «mejora» dentro de este debate no entenderemos todo tipo de intervención con fines de mejorar las habilidades y capacidades del ser humano, sino algunas de un tipo especial. Tercero, que emplear el término «mejora» en este contexto no implica referirse al alcanzar una situación más satisfactoria, sino más bien un simple intento de ello o, como lo denominé con anterioridad, una supuesta mejora. Así, una de las cuestiones esenciales será la de encontrar los criterios que debemos utilizar para delimitar qué sea eso que consideramos como mejora y qué no dentro de este debate tan específico. Algunos candidatos a ello son los conceptos: «normalidad», «natural», «terapia» 0 «modificación interior».

\subsection{Segunda dificultad: la falta de claridad de los criterios que articulan el debate}

Según lo afirmado en la sección anterior, para entrar en el debate sobre la mejora humana hemos de liberarnos de todas las connotaciones habituales que el concepto «mejora» tiene para nosotros y referirlo a un contexto especial, en el que su formulación convencional no sirve para comprender de qué se está hablando. Esta afirmación pone en duda la habitual estrategia de los autores denominados «transhumanistas», que pretende establecer una continuidad — cimentada en la percepción del «esto ya lo hemos hecho antes»— entre las intervenciones tradicionales que mejoraron nuestras capacidades —educación, instituciones, alfabetización - y las actuales en debate. A su juicio, la mejora es toda creación humana que trate de incrementar nuestras habilidades, cuerpos y mentes más allá de la media normal o habitual de la especie.

6 Debo el uso de este término a los consejos de mi tutor de tesis Jesús Conill Sancho. 
El bioético norteamericano Michael Sandel ha advertido que este deseo de ir más allá de lo que está escrito en nuestra naturaleza conlleva un afán de dominio extremadamente peligroso para nuestras sociedades 7 . Con ello, no sólo rechaza que cualquier tipo de intervención o creación humana que posea el afán de mejorar nuestra condición sea aquello a lo que se refiere este debate, sino que las intervenciones biotecnológicas con dicho afán son inaceptables moralmente. Por ello, hemos de restringir nuestro uso de las biotecnologías al ámbito terapéutico y la restauración de las capacidades normales y naturales de los seres humanos. Sin embargo, no queda claro qué sea lo normal y natural dentro de la especie, así como tampoco la línea divisoria que existe, si es que la hay, entre estas técnicas y las terapéuticas.

Apelar al concepto de «normalidad» nos obliga a tratar con otros conceptos muy problemáticos, como por ejemplo, el de «natural» y el de «especie humana», cuya naturaleza tampoco queda clara, lo cual complica aún más el debate, pues los criterios y conceptos sobre los que se articula no están claros para nada ${ }^{8}$. A juicio de Alfredo Marcos, el concepto de naturaleza humana permite aclarar la distinción entre terapia y mejora, que aparece como el criterio más ampliamente escogido para aportar luz a este debate ${ }^{9}$. Por el contrario, la filósofa española Blanca Rodríguez ha defendido recientemente la utilidad de este concepto dentro del debate y no cree en que éste tenga tanta relevancia, al menos, en lo referido a la aceptabilidad moral de estas prácticas ${ }^{10}$.

Analizando este debate a la luz de la dualidad «terapia-mejora», tal y como los defensores de esta distinción lo plantean, podríamos decir que una intervención médica es de mejora cuando queda más allá de la terapia, que sólo nos devuelve a nuestro modo de funcionar natural o normal. Para muchos, debería estar éticamente prohibido ir «más allá de la terapia» — tal y como se titula el informe sobre las intervenciones biomédicas elaborado por Leon R. Kass como director del Consejo Presidencial sobre Bioética de la Administración Bush ${ }^{11}$. Siguiendo en esta línea, Torbjörn Tännsjö, director del Centro de Bioética de Estocolmo, establece la siguiente división tripartita: intervenciones terapéuticas negativas, intervenciones terapéuticas positivas y las intervenciones de mejora humana:

- Intervenciones negativas son aquellas realizadas con el fin de curar una enfermedad o eliminar alguna incapacidad. Un ejemplo sería la toma de antibióticos para combatir una infección bacteriana.

7 M. SANDEL: The Case Against Perfection: ethics in the age of genetic engineering, Cambridge (Mass.), Belknap Press of Harvard University Press, 2007; [trad. esp. Contra la perfección, trad. por Ramón Vilá Vernis, Barcelona, Marbot, 2007.]

8 B. RodRÍGUEZ: «Sobre la relevancia moral de la distinción mejora-tratamiento» en DILEMATA: Revista Internacional de Éticas Aplicadas, n 10, 2012, pp. 307-328.

9 A. MARCos: «Filosofía de la naturaleza humana». I Simposio del CFN, École des Hautes Études en Sciences Sociales (París), 4-5 de marzo de 2010.

10 B. RodrigueZ: «Sobre la relevancia...», cit.

11 PResident's Council: Beyond Therapy: Biotechnology and the Pursuit of Happiness, Washingtong DC, Octubre. http://bioethics.georgetown.edu/pcbe/reports/beyondtherapy/beyond_therapy_final_webcorrected.pdf [Consultado el 08-08-2011]. 
- Las intervenciones positivas tratan de mejorar el funcionamiento del organismo humano para que alcance su nivel natural o normal. Un ejemplo de este tipo de prácticas puede ser el tratamiento con hormonas del crecimiento al que se somete a un niño que sufre deficiencia de la hormona de crecimiento y posee unas tasas de crecimiento fuera de lo regular.

- La intervención de mejora trata de llevar al individuo más allá del funcionamiento que es natural para su organismo. Tännsjö ofrece un ejemplo terapéutico y aceptado de mejora, a saber, el de las vacunas, cuya función es provocar la aparición de ciertos anticuerpos - que no poseemos de forma natural en nuestro cuerpo- que nos defienden ante microorganismos patógenos como la tuberculosis, la varicela, o la hepatitis. Sin embargo, se pueden encontrar otros tipos de intervenciones que se utilizan a día de hoy cuya aplicación tiene implicaciones éticas controvertidas como, por ejemplo, las inyecciones de EPO utilizadas por los deportistas para incrementar su rendimiento atlético.

Convencionalmente en nuestras sociedades democrático-liberales estamos a favor de lo que Tännsjö denominó como «intervenciones positivas» e «intervenciones negativas», mientras que aquellas intervenciones — de mejora - que van más allá de la cura directa de enfermedades, como las que se comienzan a plantear hoy en día, son altamente controvertidas, por no decir rechazadas. Puede decirse que hay dos grandes tipos de razones morales vinculadas a este rechazo: primero, aquellas relativas al sentido moral y humano de las intervenciones y, segundo, las que están relacionadas con las consecuencias de la intervención, sobre todo, a efectos de justicia social.

La tentativa de Tännsjö es loable y necesaria para este debate. Sin embargo, la tarea de definir el concepto de mejora humana no es nada sencilla pues cada intento de llevarla a cabo implica el uso de conceptos como «normalidad», «natural-artificial», «terapia», «enfermedad», «naturaleza humana» que son muy controvertidos desde un punto de vista filosófico y también médico. El propio Tännsjö se pregunta qué significa sufrir una enfermedad tratando de mostrar las diferencias que existen entre una persona sana y otra enferma. Con ello, se da cuenta, como han mostrado autores transhumanistas como Julian Savulescu, de que las fronteras entre conceptos como «mejora»y «terapia positiva» comienzan a difuminarse. Lo cual le lleva a establecer una continuidad indisoluble entre terapia y mejora que hace imposible la tarea de definir de forma clara ambas.

Ludwig Wittgenstein ya mostró en sus Investigaciones filosóficas que la definición completa y definitoria de cualquier concepto, incluso de aquellos más simples como «mesa» o «juego», es imposible. Aun aceptando la enseñanza wittgensteniana, no debe concluirse directamente que ciertas distinciones no puedan servirnos no sólo como guía práctica, sino también como forma de aclarar nuestras discusiones teóricas. El uso de conceptos y criterios teóricos, evidentemente, no está reñido con el hecho de que éstos modifiquen sus fronteras con el tiempo o dependiendo de ciertos contextos, tal y como sucede con «terapia», «enfermedad», «salud», y «mejora». Al fin y al cabo, hasta las ciencias puras sufren modificaciones conceptuales que cambian completamente el paradigma en que operan. Sin embargo, esto no hace que deban rehuir la tarea de establecer distinciones conceptuales con las que guiarse teórica y prácticamente. 
En su artículo titulado «Reproductive choice, enhancement, and moral continuum argument» Erik Malmqvist critica ${ }^{12}$, precisamente, la tendencia transhumanista de rechazar el uso de la distinción entre terapia y mejora debido a que ésta no tiene una definición completamente clara y definitoria. Por ello, este autor reclama, de forma muy acertada, la necesidad de mostrar la utilidad de dicha distinción conceptual desde un punto de vista moral. Pues, a su juicio, no es cierto que no exista diferencia moral alguna entre lo terapéutico y la mejora, de modo que la continuidad entre ambas defendida, entre otros, por la concepción bienestarista de Julian Savulescu, Anders Sandberg y Guy Kahane es falsa. Otra cosa distinta es la utilidad que pueda otorgársele teóricamente a dicha distinción. Pues el mismo autor afirma que, quizás, esta no sirva para aclarar nada sobre la aceptabilidad moral de estas técnicas, sino más bien para otras cuestiones, como, por ejemplo, tal y como explicita Blanca Rodríguez, a la hora de diseñar políticas públicas que determinen qué es aquello exigible para el Estado y qué no.

\section{Hacia una mínima definición del concepto «mejora»}

A pesar de que, como hemos visto, las dificultades sean muchas y variadas, es necesario establecer una definición, aunque sólo sea de mínimos, de aquello que consideramos como «mejora». De hecho, tantas son las controversias alrededor de dicho concepto que algunos autores han tratado de convertir en innecesaria e inútil la tarea de definirlo, por ejemplo, Leon Kaas ${ }^{13}$. Dentro de la literatura referida a la mejora, tal y como lo categorizan Julian Savulescu, Anders Sandberg y Guy Kahane en su artículo titulado «Well-Being and Enhancement», podemos encontrar cuatro posiciones diversas-y varias de ellas, curiosamente rechazan la necesidad de llevar a cabo la empresa de definir tal concepto. A saber, la pragmático-sociológica, la ideológica, la posición «no-medicamentos» y la funcional, a la que hay que añadir, evidentemente, propia de aquellos tres autores: la teoría bienestarista (welfarist).

\subsection{Las diversas posiciones}

La posición pragmático-ideológica, que incluye a autores como Erik Parens, Paul Root Wolpe o James Canton, parte de la constatación inicial de que existe una gran confusión e incertidumbre acerca de qué puede significar el concepto «mejora». La razón de esta confusión radica, según estos autores, en el origen pragmático-social de los términos que estamos obligados a utilizar para conceptualizar la mejora. Los conceptos de «enfermedad», «salud», «naturalidad», o «normalidad», se construyen histórica y culturalmente, es decir, en relación a las culturas concretas y el tiempo en que vive cada una de ellas. Así por ejemplo,

12 E. MalmQvist, «Reproductive Choice, Enhancement, and the Moral Continuum Argument» en Journal of Medicine and Philosophy [En prensa].

13 «el sentido humano y la evaluación moral deben ser abordados directamente; es poco probable que se solucionen mediante el término «mejora», como tampoco lo serán por la intervención tecnológica». PRESIDENT'S Council: Beyond Therapy: Biotechnology and the Pursuit of Happiness, New York, Regan Books, 2003 cit. por J. Savulescu, G. Kahane, A. Sandberg: «Well-Being and Enhancement», p. 4 en J. Savulescu, R. Meulen, G. KAHANE (EDS.): Enhancing Human Capabilities, London, Wiley-Blackwell, 2011, pp. 3-18. 
el concepto de salud no puede ser el mismo en un país desarrollado como España que en otro en vías de desarrollo como Marruecos, donde aún sufren enfermedades y condiciones que nuestro país dejó de tener hace 150 años. A juicio de estos autores, por lo tanto, más que un concepto único de «mejora», serán necesarios varios que se acoplen a las distintas realidades con que nos encontramos.

Para la aproximación ideológica el punto de partida es muy similar al de la expuesta anteriormente, pues se basa en del desacuerdo que, de hecho, existe en torno al concepto que se busca definir. Sin embargo, la diferencia entre ambas radica en que, simplemente, la corriente llamada ideológica evita el uso del mismo, pues no cree en la necesidad de ofrecer un concepto con el que entendernos de algún modo, aunque sea de un modo contextual y relativista. Por ello, elabora una lista de posibles técnicas de mejora y metas a las que aspira dicha tecnología, que muestra lo que hemos de entender por mejora. Más tarde, éstas se contraponen a todos aquellos valores y principios que vulnerarían su puesta en práctica. Es decir, esta posición teórica se dedica, simplemente, a clasificar las diversas técnicas una a una y calificarlas como moralmente rechazables, aceptables, o problemáticas.

La posición «no-medicina» es, sin duda, la más extendida en el debate. Es la defendida por Tännsjö y la que toman otros autores como Eric T. Juengst o Edmund D. Pellegrino. Ésta, como hemos podido comprobar más arriba, se basa en concebir la mejora como un ir más allá de los tratamientos de restauración de la salud, «yendo «más allá» de lo que existe en un momento [...] más allá de los fines de la medicina tal y como tradicionalmente se han conformado ${ }^{14} \gg$. El influyente autor Norman Daniels es también uno de sus defensores. Éste apela al modo estadístico de concebir la normalidad propuesto por Christopher Boorse, y concibe la enfermedad como «cualquier estado de la biología o psicología de una persona que reduce el funcionamiento normal típico de su especie por debajo de un nivel definido estadísticamente ${ }^{15}$ ». Entonces, la mejora es «cualquier cambio en la biología o la psicología de la persona que aumenta el funcionamiento normal típico de la especie por encima de algún nivel definido estadísticamente ${ }^{16} \gg$.

Por último, la posición funcional, al contrario que las anteriores no evita definir el concepto, ni verlo como «aquello-que-no-es-medicina», sino que lo concibe en términos de las funciones de diverso tipo que se mejoran: cognitiva, moral, física... Así, el concepto «mejora» es entendido en un sentido literal, a saber, como el incremento de cualquier tipo de habilidad humana. Esta posición aunque próxima a la anterior, defendida por Norman Daniels, se diferencia de ella en que no posee un concepto de normalidad o naturaleza que haga las veces de criterio demarcador, sino que la mejora es cualquier incremento de una habilidad humana. Así, por ejemplo, una operación terapéutica que trate de restaurar la vista de un paciente con miopía será una mejora de su vista-como veremos esta concepción tiene puntos comunes con la defendida por la teoría bienestarista.

Los miembros del Uehiro Centre for Practical Ethics de la Universidad de Oxford: Julian Savulescu, Anders Sanders, y Guy Kahane proponen una concepción bienestarista de la mejora humana que se basa, esencialmente, en una concepción consecuencialista-utilitarista

14 E.D. Pellegrino: Biotechnology, Human Enhancement, and the Ends of Medicine, The Center for Bioethics and Human Dignity, 2004, cit. por J. SAVULESCU: «Well-Being and Enhancement», cit. p. 5.

15 J. Savulescu, G. Kahane, \& A. SAndBerg: «Well-Being and Enhancement», cit., p. 4.

16 Ibíd. 
de la ética. Ésta va más allá de toda distinción problemática, ya que conceptualiza la naturaleza humana en referencia al bienestar. A juicio de esta propuesta teórica, cuando hablamos de «mejora» no lo hacemos de un simple incremento de alguna capacidad o atributo humano, sino de mejorar la vida de las personas, llevarla a un estado mejor o, en términos de la teoría de las capacidades, empoderarla con el fin de que el sujeto pueda llevar a cabo el modo de vida que tiene razones para valorar - utilizando términos propios del enfoque de las capacidades elaborado por Amartya Sen y Martha Nussbaum.

Estos autores definen «mejora» como «cualquier cambio en la biología o psicología de una persona que incrementa las posibilidades de llevar a cabo una buena vida en referencia a cuestiones relevantes ${ }^{17}$ » $\mathrm{y}$ utilizan los términos «estados mejoradores», «habilidades mejoradoras», o «estados que dan ventaja», para referirse a aquellas habilidades o capacidades que al ser mejoradas incrementan nuestras posibilidades de llevar a cabo una vida mejor. A su vez, una enfermedad es conceptualizada como «cualquier estado de la biología o psicología de una persona que reduce su capacidad para poder llevar a cabo una vida buena ${ }^{18}$ ».

La ventaja que estos autores ven en su propuesta es que no requiere la utilización de la distinción entre tratamiento terapéutico y mejora, ni del concepto «normalidad», pues al fin y al cabo todo depende, única y exclusivamente, del capacitar a los individuos para realizar sus planes de vida buena y ser con ello más felices. El bienestar es el criterio central aunque no el único, pues, por ejemplo, Julian Savulescu expondrá en otros trabajos qué otros elementos, como el incremento de las desigualdades existentes entre pobres y ricos, deben tenerse también en cuenta. Así por ejemplo, un incremento espectacular del cociente intelectual de un individuo que no influye positivamente en sus posibilidades de llevar a cabo una vida mejor no se calificaría como mejora. De igual manera, no contarían como tal ciertos intentos de intervenir en la naturaleza de los individuos que tienen consecuencias adversas para su bienestar, como por ejemplo, aumentar la capacidad auditiva de un individuo que vive en una zona muy ruidosa, por ejemplo, Manhattan.

¿Cuál es, entonces, la diferencia entre tratamientos terapéuticos, que tratan de curar a los sujetos, y aquellos que los llevan más allá de su constitución heredada? Para esta propuesta bienestarista la diferencia es simplemente de grado: el tratamiento terapéutico no es más que un tipo de mejora, al igual que las enfermedades son una subclase de discapacidad o de estado desventajoso. Así, según estos autores, existen tres grados diversos en los que puede darse la mejora — que como veremos se identifican con la división tripartita establecida por Tännsjö: tratamientos médicos de enfermedades, aumento del potencial humano natural, y mejoras sobrehumanas. Incluir el tratamiento terapéutico y la mejora en una misma categoría inseparable y gradual es fruto de lo que anteriormente denominé como estrategia por continuidad. Sus autores creen que con ello consiguen solventar gran parte de las dificultades expuestas anteriormente que surgen al tratar de analizar las cuestiones morales derivadas de este tipo de intervenciones médicas.

No obstante, esta es una propuesta muy polémica que ha sido ampliamente criticada. Así, por ejemplo, Blanca Rodríguez y Erik Malmqvist afirman que la pretensión de eliminar dicha distinción es equivocada, pues existen diferencias moralmente relevantes entre la

17 J. Savulescu: «Well-Being and Enhancement», op. cit. p. 7.

18 Ibíd. p. 7. 
terapia y la mejora. Lo cual no significa, como ambos autores advierten, que esta distinción sirva para arrojar luz sobre todas las cuestiones problemáticas que se originan en torno a la mejora. Así por ejemplo, ambos coinciden en que esta distinción no sería válida para dilucidar el tema de la aceptabilidad moral de las técnicas de mejora. Si bien la filósofa española lo afirma tajantemente, el sueco vacila respecto a esta cuestión e invita a llevar a cabo una investigación más amplia y detallada.

En lo que sí parecen coincidir los dos filósofos de forma amplia, es en que uno de los usos primordiales que debe dársele a la distinción entre terapia y mejora es el referido a la discriminación entre políticas subvencionadas por seguros de salud y aquellas que no lo serían, sobre todo a un nivel público-estatal. Algo que, por otra parte, había sugerido, a pesar de defender la tesis continuista ${ }^{19}$, John Harris en su texto Enhancing evolution: The Ethical Case for Making Better People ${ }^{20}$. Puesto que este artículo se plantea una definición global que cubra, principalmente, tanto las cuestiones de justicia social como aquellas relativas al sentido moral y humano de las intervenciones de mejora humana, prescindirá de la distinción entre terapia y mejora, aunque sin rechazarla como inservible sino, todo lo contrario, como aplicable al contexto para el que es adecuado.

\subsection{Propuesta para una definición de la mejora}

Considerando la cuestión de un modo global, no parece que los problemas éticos que se recogen bajo el término «mejora» tengan que referirse exclusivamente a aquello que está más allá del tratamiento terapéutico, sino que algunas terapias biotecnológicas, como la intervención de embriones para curar enfermedades hereditarias, también entran de forma directa dentro de este debate. Principalmente, porque lo que está en juego moralmente hablando es mucho más que el simple bienestar — que, por otra parte, es un término también muy controvertido- - a saber, la intervención técnica de aquello que hasta el día de hoy fue indisponible de un modo tan directo: nuestra naturaleza interna heredada.

Propongo definir el concepto «mejora» como intervención biomédica practicada para llevar ciertas habilidades humanas a un estado superior buscando un nivel de vida superior. Además, siguiendo la propuesta de Nicholas Agar, distingo entre dos tipos de intervenciones de mejora en función de ese estado superior al que pretendan llevar nuestras habilidades: las moderadas y las radicales. Las primeras son aquellas que no superarían ningún máximo alcanzado alguna vez por un ser humano, mientras que las segundas, por el contrario, son aquellas que excederían los límites que han sido posibles dentro del género humano ${ }^{21}$.

Cabe resaltar dos componentes esenciales de esta definición, a saber, «intervenciones biomédicas» $\mathrm{y}$ «buscando». Ya anticipé al principio de este texto qué relevancia tienen ambos elementos, pero ahora voy a explicitarlo más detalladamente — lo cual nos permitirá evaluar si esta definición es incapaz de eludir las dificultades que planteé a lo largo del

19 Un problema de los autores transhumanistas es que en ocasiones no acaban de definir el plano teórico o conceptual en que se están moviendo, pues siempre tratan de aportar una mirada demasiado global y aglutinante, así por ejemplo, a veces no queda claro si están tratando cuestiones de sentido moral y humano, o de justicia social.

20 J. HARRIS: Enhancing Evolution: The ethical case for making better people, Princenton (N.J), Princenton University Press, 2007.

21 N. Agar: Humanity's End: Why We Should Reject Radical Enhancement, London, MIT Press, 2010 , p. 17. 
apartado anterior sino, todo lo contrario, trata de amoldarse a ellas y hacerles frente. Por el momento, dejaré de lado el rol que juega la distinción tomada de Agar, que sirve principalmente para evaluar qué tipo de intervenciones pueden ser aceptables moralmente y cuáles no, un tema que está fuera de la intención de este texto.

Por un lado, resalto que es necesario referirse a intervenciones biomédicas por dos motivos principales. Primero, aún a riesgo de caer en lo que Allen Buchanan llama «excepcionalismo biotecnológico», se ofrece una definición no muy amplia de mejora, que realmente acota de forma directa y clara aquel problema al que nos estamos enfrentando — de hecho, aunque el mismo Buchanan defiende una posición continuista y una definición amplia de la mejora, va alternando ésta con una definición, llamémosla, excepcionalista. También Julian Savulescu está refiriéndose últimamente a biomejoras para abordar este debate ${ }^{22}$, a pesar de que él también es defensor de una definición amplia de la misma. Segundo, insistiendo más aún en la necesidad de enfrentarse a definiciones amplias, que dejan demasiadas cosas en el aire, la utilización del término «intervención» es clave, pues no sólo es que una intervención - técnica además - es algo muy distinto de una idea, la cultura, el Estado, o la alfabetización, sino que siempre hubo intervenciones de tipo técnico en la Historia de la Humanidad, y de hecho, algunas de ellas fueron rechazadas en base a criterios morales, incluso cuando podían incrementar el bienestar de ciertos individuos.

El hecho de que una intervención busque la mejora de la condición de los seres humanos y la consiga no es criterio suficiente para que sea aceptable moralmente. Por ejemplo, la medicina occidental sufrió un gran avance como fruto de las brutales prácticas que los médicos nazis realizaron en los judíos internados en los campos de concentración. Sin embargo, nadie puede afirmar que éstas sean correctas. Sin ir a casos tan extremos, también la extensión de la energía nuclear hizo la vida más fácil para las sociedades occidentales al proporcionar un modo económico y masivo de producción de electricidad, sin embargo, los peligros y posibles consecuencias para las futuras generaciones nos ha llevado a condenar este tipo de obtención de energía.

Por otra parte, ya en referencia al segundo término mencionado: «buscando», el término «mejora» tomado literalmente ya apunta, como afirman algunos transhumanistas, a algo positivo que lleva a un estado superior, no obstante, en realidad no es tal cosa. Como ya mostré, este término no debe comprenderse de una forma literal o «de diccionario», sino en su contexto, y éste es el de unas intervenciones biomédicas que buscan alcanzar un estado de cosas mejor para los individuos. Así, hay que aclarar que una cosa es buscar un estado mejor, y otra muy distinta es que ese estado, de hecho, se consiga. Bien puede ser que una pretendida mejora acabe resultando ser una peora.

Esto no lo puede decidir el significado del término, sino un análisis detallado y particular de los modos de mejora y los contextos en que son aplicados. Así por ejemplo, una mejora física genéticamente producida, al estilo de los famosos superratones que más tarde mencionaré, podría ser beneficiosa para un sujeto que vaya a ser un soldado, por contra, ésta será negativa en un futuro deportista, pues, ante las actuales normas anti-dopaje, se le negaría su participación en cualquier tipo de competición deportiva de élite. Siempre existieron criterios

22 J. Savulescu \& I. Persson: Unfit for the Future? The need for moral enhancement, Oxford, Oxford University Press, 2012. 
éticos para evaluar las diversas intervenciones técnicas humanas, por ejemplo, se distingue entre modos de educación aceptables y aquellos que no lo son, al igual que, en otro orden de cosas, también se rechazaron las prácticas eugenésicas estatales de principios del $\mathrm{S}$. XX.

Este «buscar llevar a los individuos a un estado de cosas mejor», tomado de la posición bienestarista, resulta muy interesante, pues conduce a la definición de mejora más allá de lo descriptivo para situarla en el ámbito de lo normativo. Así, ésta recibe sentido en ese trasfondo moral que supone la capacidad de poder llevar a cabo una vida buena. En este terreno es en el que puede librarse la batalla moral sobre la aceptabilidad o no de estas técnicas, así como la de su exigibilidad. Y en el que, por cierto, encaja la distinción de Nicholas Agar. De este modo, mi definición de mínimos sirve para acotar el problema. Además, al contrario que muchos autores transhumanistas, ésta no rechaza el uso de distinciones complementarias que serán muy útiles para solucionar problemáticas más concretas. Entre estas distinciones puede incluirse, por supuesto, la establecida entre mejora y terapia para, en claro acuerdo con Blanca Rodríguez, decidir qué es aquello exigible y qué no en relación a la mejora con respecto, sobre todo, a nuestros Estados sociales de derecho.

Si bien esta deriva normativa de la teoría bienestarista es un punto a su favor muy importante, cabe hacerle algunas críticas. Por ejemplo, el hecho de que afirme que toda teoría bienestarista, independientemente de los criterios con que evalúe el bienestar, estaría de acuerdo con sus premisas. No puedo elaborar aquí una exposición de las teorías bienestaristas para comprobar o falsar esta afirmación — de hecho, ni los propios defensores de esta teoría llevan a cabo tal tarea de forma exhaustiva. El problema principal radica en que con ello se evita, de nuevo, la definición de qué se considera como bienestar, qué criterios lo miden, y cómo las intervenciones biomédicas incrementan ese bienestar. Tampoco ésta es una tarea que quepa hacer aquí, sin embargo, sí trataré de llevarla a cabo en futuros trabajos. Para concluir esta formulación de mínimos del concepto «mejora» explicaré cuáles son los tipos de intervenciones que cuadrarían con ella y, a groso modo, cuál es el estado actual en que se encuentra su desarrollo. Sólo así sabremos completamente a qué nos enfrentamos cuando entramos en este debate bioético.

\section{Tipos de mejoramiento, ¿qué es posible y qué no?}

Suelen diferenciarse cinco tipos de mejoramiento ${ }^{23}$ : cognitivo, físico, moral, anímico y el referido a la extensión de la vida. Como indica Allen Buchanan ${ }^{24}$, aunque los métodos para llevarlos a cabo dependen de la especificidad de cada uno, pueden encontrarse cinco modos de terapia comunes a todos:

1. la selección genética de embriones para su posterior implantación.

2. la intervención y modificación genética de embriones.

3. la administración de drogas.

23 N. Bostrom, \& R. Roache: «Ethical Issues in Human Enhancement» en J. Ryberg (Ed.): New Waves in Applied Ethics, Virginia (USA), Palgrave MacMillan, 2008. http://www.nickbostrom.com/ethics/human-enhancement.pdf [Consultado el 08-08-2011].

24 A. Buchanan: Beyond Humaniy?, cit., p. 25. 
4. la implantación de tejidos, material u órganos genéticamente modificados.

5. implantación de interfaces cerebro-ordenador.

\subsection{Mejora Cognitiva ${ }^{25}$}

El mejoramiento cognitivo puede ser definido como «la amplificación o extensión de las capacidades centrales de nuestra mente mediante el aumento o incremento de nuestros sistemas de procesamiento de información ${ }^{26} \gg$. Para algunos, como ya se ha indicado, la educación o el entrenamiento mental pueden ser concebidos como métodos de mejoramiento que incrementan nuestra capacidad de procesar información. Sin embargo, estos procedimientos serán los que denominemos «convencionales» por el hecho de que no son moralmente polémicos. Los que aquí nos interesan son aquellos que podríamos denominar como «no-convencionales», es decir, los de naturaleza biotecnológica, que son los que presentan recelos y generan preocupación ante la opinión pública.

\subsection{Mejoramiento anímico ${ }^{27}$}

La introducción del Prozac marcó el inicio del desarrollo de este tipo de prácticas, ya que su surgimiento provocó la aparición de una gran cantidad de inhibidores selectivos de recaptación de la serotonina (ISRS) que conforman «los nuevos antidepresivos». Los ISRS aumentan los niveles extracelulares del neurotransmisor serotonina, inhibiendo su recaptación e incrementando el nivel disponible de serotonina. Inicialmente este tipo de tratamientos estaban reservados a casos de depresión o desórdenes afectivos que son reconocidos como enfermedades mentales, sin embargo, algunos de estos «mejoradores del ánimo» están prescribiéndose a personas sin ningún tipo de síntomas de enfermedad mental, en los que el funcionamiento de su cerebro es completamente normal, simplemente, con el fin de «sentirse mejor que bien», más alerta, más enérgicos, más capaces de enfrentarse al mundo y a sus problemas ${ }^{28}$.

\subsection{Mejoramiento físico}

Las intervenciones de mejora física más comunes actualmente son el tratamiento con esteroides, la ingestión de suplementos nutricionales, vitaminas y alimentos funcionales, la cirugía selectiva, el tratamiento con factores de crecimiento y hormonas, la modulación del dolor, y los tratamientos a través de la sangre. Dentro de este tipo de mejoras ya pueden incluirse ciertos tipos de tratamientos de naturaleza biomédica, concretamente genética.

25 A. Sandberg: «Cognition Enhancement: Upgrading the Brain» en J. Savulescu, R. Meulen, G. Kahane (EDs.), Enhancing Human Capabilities, Oxford, Balckwell Publishing, 2011, pp. 71-91.

26 Ibíd. p. 71.

27 R. Berghmans, A. Meulen, A. Malizia, \& R. Vos: «Scientific, Ethical, and Social Issues in Mood Enhancement» en J. Savulescu, R. Meulen, G. Kahane (Eds.): Enhancing Human Capabilities, cit., pp. 153-165.

28 C. Elliot: Better than Well: American Medicine Meets the American Dream, W.W. Norton, New York, 2003 cit. por R. Berghmans, R. Meulen, A. Malizia, \& R. Vos, op. cit. 
Por ejemplo, el uso de Eritropoyetina (EPO) — una potente hormona producida en los riñones que regula la cantidad de oxígeno que puede transportar la sangre aumentando el número de glóbulos rojos. También se plantea la posibilidad de tratamientos genéticos que consistan en modificaciones genéticas previas al nacimiento o incluso somáticas en adultos que permitan, por ejemplo, el crecimiento de los músculos por encima de un ratio normal, tal y como se ha mostrado en el famoso experimento de los superratones llevado a cabo por el equipo de Se-Jin Lee ${ }^{29}$, que descubrió la relación entre dos proteínas llamadas miostatina y folistatina.

\subsection{Extensión de la vida 30}

Cinco vías de tratamiento — o mejoramiento— de la duración de la vida están comenzando a investigarse:

1) La restricción de la toma de calorías complementada con la ingesta del adecuado nivel de proteínas, vitaminas, minerales y otros nutrientes. Este tipo de experimentos, que ha tenido éxito en ratones, ya ha sido llevado a seres humanos, demostrando que una dieta de este tipo tiene consecuencias beneficiosas para varias de sus funciones fisiológicas. Sin embargo, ni se ha demostrado que tengan influencia en el proceso de envejecimiento, ni parece una solución viable debido a los posibles efectos secundarios — hipotensión, infertilidad, osteoporosis, depresión, irritabilidad. El reto está en encontrar un modo de tratamiento artificial que tenga los mismos efectos y que no influya sobre la dieta de los individuos.

2) Tratamientos hormonales mediante hormonas del crecimiento, factor de crecimiento insulínico de tipo 1 (IGF-1), melatonina, testosterona, prosgesterona o estrógenos. Por ejemplo, el uso de hormonas del crecimiento ha mostrado efectos positivos para patologías derivadas del envejecimiento, ayudando a frenar la pérdida de masa muscular o de densidad mineral ósea. Sin embargo, tampoco existen pruebas de su incremento de la duración de la vida.

3) Limitar los procesos degenerativos de las células reduciendo los daños provocados por la oxidación o actividad telomerásica.

4) Aunque no parezcan existir genes concretos causantes del envejecimiento, se están buscando algunos que puedan tener efectos sobre algunos de los procesos antes mentados, con el fin de llevar a cabo intervenciones genéticas que amplíen la duración de nuestras vidas. Sobre todo, aquellos relacionados con los mecanismos encargados del mantenimiento y reparación de las células. En este sentido, se ha mostrado que ratones con p66ShcA deficiente son más resistentes al estrés de oxidación, por lo que viven más que el resto.

29 R.M. TRISTÁN: «Crean «superratones» cuatro veces más musculosos que los normales» en Diario El Mundo, 30-08-2007. http://www.elmundo.es/elmundo/2007/08/30/ciencia/1188461613.html [Consultado el 10-092011].

30 G. BARAZZETTI: «Looking for the Fountain of Youth. Scientific, Ethical and Social Issues in the Extensión of Human Lifespan» en R. Berghmans, R. Meulen, A. Malizia \& R. Vos: op. cit., pp. 335-349. 
5) Por último, no pueden olvidarse las aplicaciones tecnológicas, sobre todo, las relacionadas con las células madre utilizadas con el fin de reemplazar tejidos dañados de los órganos.

\subsection{Mejoramiento moral $^{31}$}

El mejoramiento moral es, quizás, el campo menos avanzado experimentalmente de todos, sobre todo, por la dificultad que tiene establecer la relación que existe entre nuestras bases biológicas y la creación humana que supone la moral. A pesar de ello, sí existen diversos estudios relacionados con ciertas emociones como la agresividad o la aversión racial, que muestran la correlación de comportamientos influidos por ellas y, por ejemplo, ciertas zonas del cerebro - como sucede con la amígdala y la aversión racial- o genes. Sin embargo, parece que no hemos de esperar a un corto o medio plazo avances de verdadera importancia en este campo ${ }^{32}$.

\section{Conclusión: una concepción moral del mejoramiento humano}

Recapitulando lo mostrado, podemos concluir que, si bien muchos autores acaban decantándose por una definición continuista y amplia de la mejora, este artículo propone una más específica, la cual reduce este término a aquel tipo de intervenciones biomédicas que se realizan con la finalidad de mejorar la vida de las personas (§3). Esta definición no deja de lado distinciones y conceptos fundamentales para la discusión en torno a la mejora, sino que las reserva para contextos concretos. Como sucede con el caso de la distinción entre terapia y mejora y el caso de aquello que es exigible a un sistema de seguros sanitarios, bien sean públicos o privados.

De este modo, se evitan dificultades propias de este debate que no hacen más que complicar el hecho de que podamos identificar claramente aquello a lo que nos referimos cuando hablamos de intervenciones de mejora (\$2). Por ello, este artículo concluye mostrando que existen cinco tipos de intervenciones de mejora, a saber: cognitivas, del carácter, físicas, relativas al envejecimiento, y morales $(\$ 4)$.

Aunque aportar un concepto claro de mejora es un gran paso, ha quedado demasiado por hacer en este trabajo. Muchas han sido las promesas teóricas que he reservado para el futuro. Entre ellas, querría resaltar esa propuesta de la teoría bienestarista que nos invita a considerar la cuestión de la mejora dentro de un marco esencialmente normativo: la capacidad que los individuos tienen de alcanzar los modos de vida que valoran como buenos.

Como afirmaba el filósofo español José Luis Aranguren, somos seres constitutivamente morales, lo que exige esta inevitable constitución moral es que la técnica no se haga dueña del hombre, sino que sea él mismo quien la maneje de la mejor manera posible con el fin de incrementar las oportunidades que tiene para llevar a cabo vidas mejores. Como buena discípula de Aranguren, Adela Cortina, apelando a la célebre novela de Mary Shelley Frankenstein, afirma respecto a la mejora que: «los miembros y los órganos de un ser humano, incluido el cerebro, pueden ser muy perfectos, pluscuamperfectos, pero nada garantiza que

31 T. Douglas: «Moral Enhancement» en J. Savulescu, R. Meulen, G. Kahane (Eds.): Enhancing Human Capabilities, cit., pp. 467-485.

32 J. Savulescu \& I. Persson, «Moral Enhancement» en Philosophy Now, 91, 2012. http://philosophynow.org/ issues/91/Moral_Enhancement [Consultado el 29-11-2012]. 
su vida sea una vida buena si no puede contar con otros entre los que saberse reconocido y estimado ${ }^{33}$ ». Si no insertamos la reflexión sobre las técnicas de mejora en el contexto de la vida buena, éstas pierden su sentido completamente.

\section{Bibliografía}

\section{Libros}

AGAR, N. Humanity's End: Why we should reject radical enhancement, Cambridge (Mass.), MIT Press, 2010.

BAYERTZ, K. GenEthics: technological intervention in human reproduction as a philosophical problema, Cambridge, Cambridge University Press, 1994.

BEAUCHAMP, T.F. \& CHILDRESS J.F. Principles of Biomedical Ethics, Oxford University Press, Oxford, 1979 [trad. cast. por Rafael Herrera Bonet, Principios de ética biomédica, Barcelona, Masson, 1999].

BUCHANAN, A. Beyond Humanity? The Ethics of Biomedical Enhancement, Oxford University Press, Oxford, 2010.

CORTINA, A. Ética de la razón cordial, Ediciones Nobel, Oviedo, 2007.

- Las fronteras de la persona, Taurus, Madrid, 2009.

ERHARD, J.B., HERDER, J.G., KANT, I., ET AL: ¿Qué es la Ilustración?, trad. por Agapito Maestre, Tecnos, Madrid, 1988.

FUKUYAMA, F. Our Posthuman Future: Consequences of the Biotechnology Revolution, Profile Books, London, 2002 [trad. cast. El fin del hombre: consecuencias de la revolución biotecnológica, trad. por Paco Reina, Zeta Bolsillo, Barcelona, 2008].

GLOVER, J. What Sort of People Should There Be? Genetic Engineering, Brain Control and Their Impact on Our Future World, New York, Penguin Books, 1984 [trad. esp. El hombre prefabricado: problemas éticos de la ingeniería genética, Barcelona, Ariel, 1986].

HARRIS, J. Enhancing Evolution: The ethical case for making better people, Princeton (N.J), Princeton University Press, 2007.

NAAM, R. More Than Human, Broadway Books, New York, 2005.

SANDEL, M. The Case against Perfection: Ethics in the Age of Genetic Engineering, Harvard University Press, Cambridge, 2007 [trad. cast. Contra la perfección: La ética en la época de la ingeniería genética, trad. por Ramón Vilá Vernis, Marbot Ediciones, Barcelona, 2007].

SAVULESCU, J. \& BOSTROM, N. (EDS.) Human Enhancement, Oxford University Press, Oxford, 2009.

SAVUlESCU, J., TEER MEULEN, R. \& KAHANE, G. (EDS.) Enhancing Human Capacities, Wiley-Balckwell, Oxford, 2011.

33 A. CORTINA: «Frankenstein: el origen de la Neuroética» en Diario El País, 17-10-2010. http://www.elpais.com/ articulo/opinion/Frankenstein/origen/Neuroetica/elpepiopi/20101017elpepiopi_5/Tes [Consultado el 10-082011]. 


\section{Artículos}

ALLHOFF, F. LIN, P. MOOR, J. \& WECKERT, J. «Ethics of Human Enhancement: 25 Questions \& Answers» en Studies in Ethics, Law, and Technology, Vol. 4 Nº1, Berekeley Electronic Press, 2010.

BOSTROM, N. \& ROACHE, R. «Ethical Issues in Human Enhancement» en J. RYBERG (ED.), New Waves in Applied Ethics, Virginia (USA), Palgrave MacMillan, 2008.

CAPLAN, A.L. "An unnatural process: Why is not inherently wrong to seek or cure for aging» en S.G. POST \& R.H. BINSTOCK (EDS.), The Fountain of Youth. Cultural, Scientific, and Ethical Perspectives on a Biomedical Goal, Oxford University Press, Oxford, 2004, pp. 271-285.

COECKELBERGH, M. «Human development or human enhancement? A methodological reflection on capabilities and the evaluation of information technologies» en Ethics and Information Technologies, 13, vol. 2, pp. 81-92.

CORTINA, A. «Frankenstein: el origen de la Neuroética» en Diario El País, 17-10-2010.

FOSTER, J.K., LIDDER, P.G, \& SUNRAM, S.I «Glucose and Memory; fractionation of enhancement effects?» en Psycopharmacologhy, 137, vol. 3, pp. 259-270.

GLADSTONE, D.J. \& BLACK, S.E. «Enhancing recovery after stroke with noradrenergic pharmacotherapy: A new frontier?» en Canadian Journal of Neurological Sciences, 27, vol. 2, pp. 97-105.

GONZÁLEZ, M. «La genética personal topa con la patente» en Diario El País, 31-03-2011.

MARCOS, A. «Filosofía de la naturaleza humana». I Simposio del CFN, École des Hautes Études en Sciences Sociales (París), 4-5 de marzo de 2010.

PARENS, E. «Is better always good? The Enhancement Project» en E. PARENS (ED.): Enhancing Human Traits: Ethical and Social Implications, Georgetown, Georgetown University Press, 1998.

RAE, A. et al: "Oral creatine monohydrate supplementation improves brain performance: a double-blind, placebo-controlled, cross-over trial» en Procedings of the Royal Society of London Series B, Biological Sciences, 270 (1529), pp. 2147-50.

RODRÍGUEZ, B. «Sobre la relevancia moral de la distinción mejora-tratamiento» en DILEMATA: Revista Internacional de éticas aplicadas, 4 (2012), 10, pp. 307-328.

TANG, Y.P. et all «Genetic enhancement of learning and memory in mice» en Nature, 401 (6748), pp. 63-69. 
